\title{
Reliable Routing Algorithm for Wide Area Protection
}

\author{
Chuan $\mathrm{Liu}^{1,2}$, Jing Tao ${ }^{1,2}$, Zheng $\mathrm{Lou}^{3}$ and Qi Shao ${ }^{4}$ \\ ${ }^{1}$ Global Energy Interconnection Research Institute Co,. Ltd, Nanjing, China \\ ${ }^{2}$ State Grid Laboratory of Electric Power Communication Network Technology, Nanjing, China \\ ${ }^{3}$ Nanjing Power Supply Company, State Grid Jiangsu Electric Power Company, Nanjing, China \\ ${ }^{4}$ State Grid Henan Information \& Telecommunication Company, Zhengzhou, China
}

\begin{abstract}
The wide area protection system has strict requirements on the real-time communication, reliability and bandwidth. How to select the optimal path quickly and efficiently is very important for the transmission of protection and control information. In order to solve this problem, a mathematical model of optimal routing is established in the paper to meet the requirements of reliability, delay and bandwidth based on graph theory. Depth-first search and Floyd algorithm are also used to solve the problem. The simulation results show that the proposed algorithm can solve the most reliable routing satisfying the delay requirement of wide area protection system, which not only improves the reliability of information transmission in wide area protection communication network, but also guarantees the realtime performance of signal transmission.
\end{abstract}

Keywords—wide area protection; routing; reliability; time delay; bandwidth

\section{INTRODUCTION}

Building on the capabilities and architecture of wide area protection, building high speed, bi-directional, real-time, selfhealing, secure and reliable communications networks is the foundation for wide-area protection [1]. Compared with the traditional monitoring and control system, wide area protection system puts forward higher requirements on the real-time and reliability of communication. Routing is the key to real-time and reliable information transmission. Especially when the power grid fails, the information flow instantly increases dramatically[2]. It is very important to choose the proper path to transmit the information quickly and reliably to the control center.

In the current Internet communication network architecture, the routing algorithm adopts the "best-effort" forwarding strategy. Dijkstra algorithm is often used to solve the path with a single constraint index such as the minimum path length and the minimum delay[3]. It does not take into account the realtime when the path forwarding and reliability, so routing algorithms under the Internet communication architecture and can not meet the requirements of wide area protection communication system. At present, research on routing of wide area communication system in smart grid is still rare. In the available literature, the related routing algorithms often do not apply to wide area protection scenarios[4][5][6][7].Based on the requirements of reliability, real-time and transmission bandwidth of wide area protection communication, this paper establishes a mathematical model of optimal routing to meet the requirements of reliability, delay and bandwidth based on graph theory and uses depth-first search Solve with Floyd algorithm. The simulation results show that the proposed algorithm can solve the most reliable routing satisfying the delay requirement of wide area protection system, which not only improves the reliability of information transmission in wide area protection communication network but also guarantees the real-time performance of signal transmission.

\section{MATHEMATICAL MODEL OF ROUTING}

In power communication network, switching equipment installed in power plants, substations, and dispatch centers are abstracted into a node set $V$, the communication channels for connecting nodes are abstracted into a link set $E$, and the restriction conditions between them are abstracted into set $C$. According to the graph theory, the topology of the final composition network satisfies $G=(V, E, C)$.

\section{A. Information Transmission Delay}

Optical fiber is the communication medium of wide area protection system. The known information propagates in optical fiber at the speed of light. Delay of path is the time needed to protect the control signal from the source node to the destination through the path. Path delay is mainly measured by the following factors: path length, information transmission speed, number of nodes, switching delay and maximum bandwidth. Information transmission delay can be expressed as the following formula which $l_{e_{k}}$ indicates the length of each link in the path, $2 / 3 \mathrm{c}$ is the propagation speed of information in the optical fiber, $n_{\text {is }}$ the total number of nodes in path $p_{i}, t_{v}$ is the exchange delay at each node, $\Delta t$ represents the delay due to unforeseen random jitter.

$$
T_{p_{i}}=\sum_{k=1}^{n-1} \frac{l_{e_{k}}}{\frac{2}{3} c}+n t_{v}+\Delta t
$$

\section{B. Path Reliability}

The shortest path is not necessarily the best path for the transmission of information. Since a fault occurs frequently in a wide area network, it is necessary to select a path with as few faults as possible for transmission control protection information. Therefore, find a road from the specified source node $\mathrm{S}$ to the destination $\mathrm{T}$ that maximizes the reliability, which is the largest reliable route from $\mathrm{S}$ to $\mathrm{T}$. 
Suppose that path $p_{i}$ is any path from $\mathrm{S}$ to $\mathrm{T}$ in wide area protection communication network. The path is formed by the link $\left\{e_{1}, e_{2}, \ldots, e_{n}\right\}$ connecting in turn, and the availability on the link is $\left\{A_{i_{1}}, A_{i_{2}}, \ldots, A_{i_{j}}, \ldots, A_{i_{n}}\right\}$ respectively. Thus, the reliability expression of the path $p_{i}$ can be represented as the following formula which $\mathrm{n}$ is the number of links that make up path $p_{i}$, $A_{i_{j}}$ is the availability of the link between node ${ }^{v_{i}}$ and node ${ }^{v_{j}}$ in path $p_{i}$.

$$
R_{p_{i}}=\prod_{j=1}^{n} A_{i_{j}}
$$

The reliability of a link needs to be calculated over a long period of operation time, and its reliability is difficult to obtain. Therefore, the reliability of a link is hard to calculate for a practical communication system. Generally speaking, the wider the path bandwidth, the lower the probability of path congestion. The more the path hops, the lower the path reliability.

\section{Path Bandwidth}

In wide area communication network, not only the equipment failure can cause information misinformation or loss, but also the unbalanced distribution of traffic can lead to the network being unable to communicate with each other. Therefore, whether the finally selected communication path has enough bandwidth will also affect whether this path can meet the requirements of communication performance. Suppose that path $p_{i}$ consists of link $\left\{e_{1}, e_{2}, \ldots, e_{n-1}\right\}$, and real-time measurement of link bandwidth is set $\left\{c_{e_{1}}, c_{e_{2}}, \ldots, c_{e_{n-1}}\right\}$. The bandwidth of path $p_{i}$ should be the minimum bandwidth of all the links that the path passes, that is, the maximum throughput of this path. The formula is as following which ${ }^{C_{i j}}$ indicates link $v_{i} v_{j}$ on link $p_{i}$.

$$
C_{p_{i}}=\min \left\{C_{i j}\right\}
$$

\section{EstABLishment OF MATHEMATICAL MODEL OF ROUTING}

\section{A. Model Establishment}

According to the above description, the routing algorithm is the most reliable routing algorithm under the conditions of wide area protection delay and bandwidth. The mathematical model of routing is as following which $N_{p_{i}}$ is the hop count for path $p_{i}, C_{0}$ indicates the minimum bandwidth that the entire road is allowed to transmit information, $C_{1}, C_{2}, \ldots, C_{k}$ Indicates the bandwidth required for all traffic transmitted across the road, $T_{0}$ Indicates the maximum delay allowed for transmission of a protection control signal over a wide area protection communication system.

$$
\begin{cases} & \left.\max \left\{C_{0} / N_{p_{i}}\right)\right\} \\ \text { s.t. } & C_{p_{i}}>\max \left(C_{1}, C_{2}, \ldots, C_{k}\right) \\ & \left(T_{p_{1}}, T_{p_{2}}, \ldots, T_{p_{i}}, \ldots, T_{p_{n}}\right)<T_{0}\end{cases}
$$

If a path satisfies $T_{p_{i}}>T_{0}$ or $C_{p_{i}}<\max \left(C_{1}, C_{2}, \ldots, C_{k}\right)$, the path is not deleted according to the delay or bandwidth requirements in this model, otherwise the path is reserved for the candidate path.

In order to control the power system in real time, the realtime information extraction, transmission and processing must be completed within $30 \sim 50 \mathrm{~ms}$ to ensure complete status information and take corresponding measures before instability or collapse. Suppose there is a provincial power grid with a scale of 50 nodes and sub-stations within a distance of $1000 \mathrm{~km}$. The transmission delay needs to be less than $20 \mathrm{~ms}$ in order to meet the communication performance requirements. Therefore, the network size is different, delay requirements are different, according to the specific circumstances set.

\section{B. Steps of Route Solving}

According to the mathematic model of routing information in WAN communication proposed in the previous section, we can see that this model is a problem that satisfies the maximization of objective function with nonlinear constraints. In this paper, the depth-first search algorithm and the improved Floyd algorithm are used to solve the model. Specific algorithm steps are shown in Figure I. 


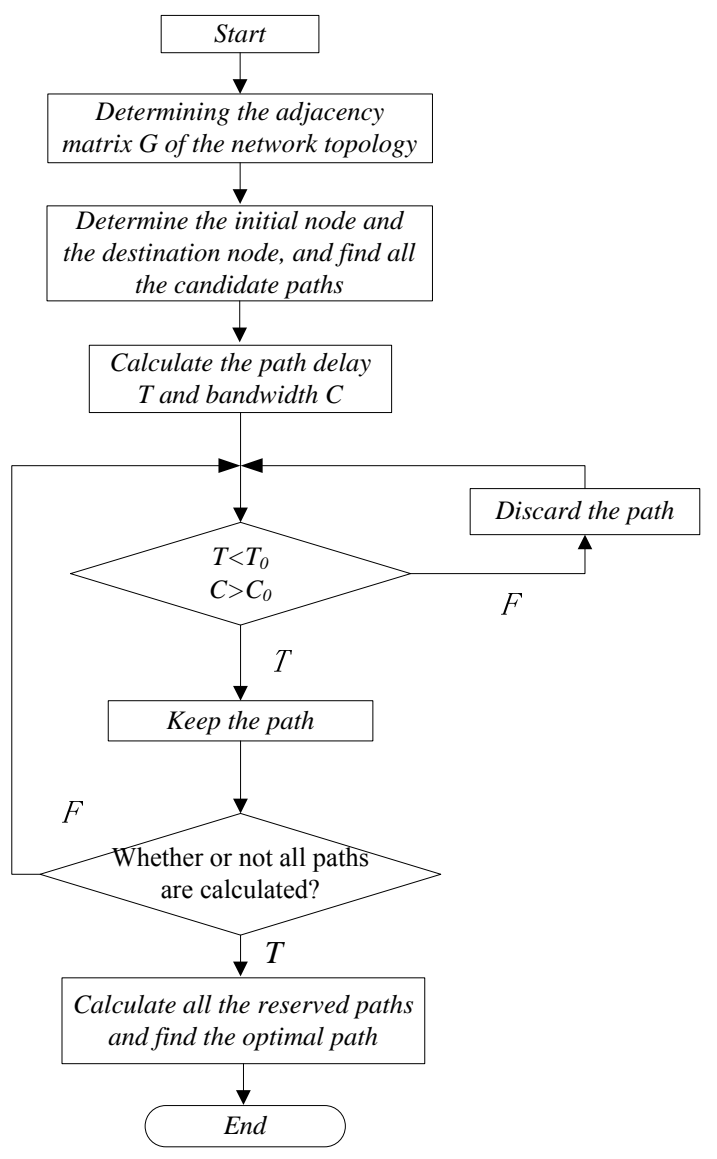

FIGURE I. ROUTING ALGORITHM FLOW CHART

The above algorithm can find the optimal path to meet the mathematical model established in this article, and calculate the maximum reliability path is the difficulty of this model. The steps that need attention in this calculation process are described in detail below.

\section{ALGORITHM AND RESULT ANALYSIS}

\section{A. Algorithm Introduced}

In order to verify the application of the model and algorithm in the wide area communication system, the network topology shown in Figure II below is used as an example to verify the analysis. In this topology, the nodes represent substations, plant sites and dispatch centers, etc. The links represent the communication channels and the communication medium is optical fiber. Since the OPGW optical network has the same topology as the power grid, the link length is the length of the power transmission line and the link and node availability parameters can be calculated from the statistics, which are given in this paper. The speed of light is $c=3 \times 10^{8} \mathrm{~m} / \mathrm{s}$, and the propagation speed of information in the optical fiber is $2 / 3 \mathrm{c}$. Unexpected random jitter generated delay $\Delta t$ take $0.1 \mathrm{~ms}$, each node exchange delay of $1 \mathrm{~ms}$.

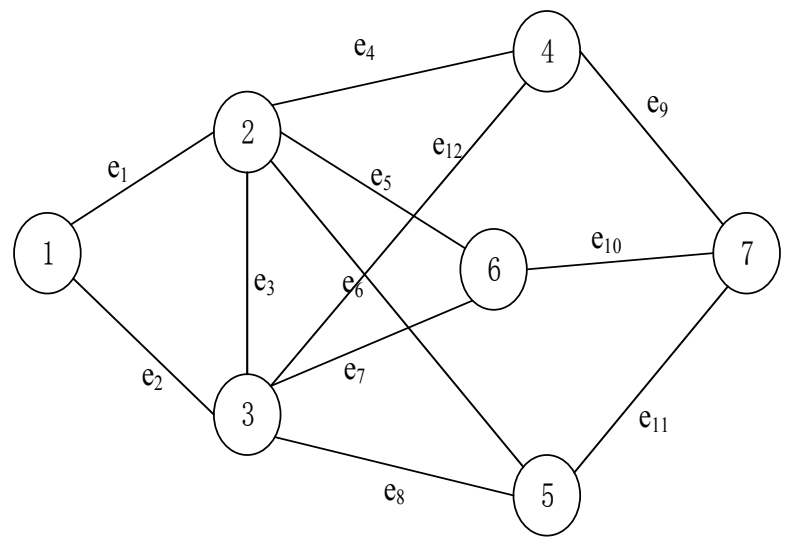

FIGURE II. NODE NETWORK

The adjacency matrix of graph $\mathrm{G}$ can be obtained from the routing algorithm, denoted as $X=\left[x_{i j}\right]_{n \times n} . \mathrm{X}$ is an n-order square matrix, $i$ and $\mathrm{j}$ are nodes, element ${ }^{X_{i j}}$ is defined by the following formula.

$$
X_{i j}= \begin{cases}0 & i=j \\ 1 & \text { There is a link between } i \text { and } j \\ 0 & \text { There is no link between } i \text { and } j\end{cases}
$$

For the connected graph in Figure I, its topological point distance adjacency matrix is shown in the following formula.

$$
\mathrm{W}=\left[\begin{array}{ccccccc}
0 & 74 & 97 & \text { inf } & \text { inf } & \text { inf } & \text { inf } \\
74 & 0 & 102 & 155 & 146 & 132 & \text { inf } \\
97 & 102 & 0 & 157 & 119 & 151 & \text { inf } \\
\text { inf } & 155 & 157 & 0 & \text { inf } & \text { inf } & 122 \\
\text { inf } & 146 & 119 & \text { inf } & 0 & \text { inf } & 128 \\
\text { inf } & 132 & 151 & \text { inf } & \text { inf } & 0 & 117 \\
\text { inf } & \text { inf } & \text { inf } & 122 & 128 & 117 & 0
\end{array}\right]
$$

The other data in the network topology, such as the availability of each node, the length of each link and availability data can be used to represent the adjacent matrix. The availability of each node in the network is marked in the topology. For the parameters of each link is shown in Table I. 
TABLE I. NETWORK LINK PARAMETERS

\begin{tabular}{|c|c|c|}
\hline Link & Length(km) & Bandwidth(Mbps) \\
\hline $\mathrm{e}_{1}$ & 74 & 5 \\
\hline $\mathrm{e}_{2}$ & 97 & 8 \\
\hline $\mathrm{e}_{3}$ & 102 & 7 \\
\hline $\mathrm{e}_{4}$ & 155 & 6 \\
\hline $\mathrm{e}_{5}$ & 132 & 2 \\
\hline $\mathrm{e}_{6}$ & 146 & 8 \\
\hline $\mathrm{e}_{7}$ & 151 & 8 \\
\hline $\mathrm{e}_{8}$ & 119 & 7 \\
\hline $\mathrm{e}_{9}$ & 122 & 9 \\
\hline $\mathrm{e}_{10}$ & 117 & 6 \\
\hline $\mathrm{e}_{11}$ & 128 & 7 \\
\hline $\mathrm{e}_{12}$ & 157 & 5 \\
\hline
\end{tabular}

Assuming that the source node 1 and the destination node 7 in the communication network need to transmit the power grid information, the optimal path is calculated by the above algorithm. The network belongs to a communication network with a size of 7 nodes and a sub-station which is within a range of $200 \mathrm{~km}$ from the mid-range. Therefore, the communication delay threshold can be set to $5 \mathrm{~ms}$, and the bandwidth threshold can be set to $4 \mathrm{Mbps}$ according to the network link parameters. Transmission in the network topology. The required bandwidth for business traffic is $4 \mathrm{Mbps}$.

\section{B. Result Analysis}

According to the routing method in this paper, the information transmission path between communication source node 1 and destination node 7 is selected. From the simulation results, a total of 24 paths are available for transmitting information in this topology network. Nineteen paths are deleted because the forwarding nodes do not meet the delay requirement of more than 4 and the path through link e5 does not meet the bandwidth requirement. By the above formula, we can see that the delay and bandwidth of the remaining five paths are both meet the model requirements, as a candidate path, the specific calculation results in Table II.

TABLE II. RESULTS OF CANDIDATE PATH

\begin{tabular}{|c|c|c|c|}
\hline $\begin{array}{c}\text { Candidate } \\
\text { Path }\end{array}$ & $\begin{array}{c}\text { Time Delay } \\
(\boldsymbol{m s})\end{array}$ & $\begin{array}{c}\text { Bandwi } \\
\mathbf{d t h}(\mathbf{M b} \boldsymbol{b} \\
\mathbf{p s})\end{array}$ & $\begin{array}{c}C_{0} *_{\mathbf{p}_{\mathbf{s}}} \mathbf{S} \\
\text { ort }\end{array}$ \\
\hline $1 \rightarrow 2 \rightarrow 4 \rightarrow 7$ & 4.1018 & 5 & 5 \\
\hline $1 \rightarrow 2 \rightarrow 5 \rightarrow 7$ & 4.1017 & 5 & 4 \\
\hline $1 \rightarrow 3 \rightarrow 4 \rightarrow 7$ & 4.1018 & 8 & 1 \\
\hline $1 \rightarrow 3 \rightarrow 5 \rightarrow 7$ & 4.1017 & 7 & 2 \\
\hline $1 \rightarrow 3 \rightarrow 6 \rightarrow 7$ & 4.1018 & 6 & 3 \\
\hline
\end{tabular}

The route chosen by the network after this chapter is $1 \rightarrow 3 \rightarrow 4 \rightarrow 7$. As shown in Table II, there are five paths satisfying the delay and bandwidth requirements between node 1 and node 7 . Among these candidate paths, the paths with the shortest delay are $1 \rightarrow 2 \rightarrow 5 \rightarrow 7$ and $1 \rightarrow 3 \rightarrow 5 \rightarrow 7$, with time of $4.1017 \mathrm{~ms}$. The remaining bandwidth of the path is $5 \mathrm{Mbps}$ and $7 \mathrm{Mbps}$ respectively, and the reliability is 0.9737 and 0.9746 respectively. The two paths have similar delay with other paths but the reliability and bandwidth are lower than $1 \rightarrow 3 \rightarrow 4 \rightarrow 7$ reliability and bandwidth. The delays of paths $1 \rightarrow 2 \rightarrow 4 \rightarrow 7$ and $1 \rightarrow 3 \rightarrow 6 \rightarrow 7$ are the same as those of paths $1 \rightarrow 3 \rightarrow 4 \rightarrow 7$, but the reliability and bandwidth are significantly lower than those of the other four paths. Among all the paths, the paths with the highest bandwidth and the highest reliability are paths
$1 \rightarrow 3 \rightarrow 4 \rightarrow 7$, which are respectively $8 \mathrm{Mbps}$ and 0.9759 , and the propagation delay of the path is very different from other paths. By using the mathematical model in this chapter and the routing method. The path $1 \rightarrow 3 \rightarrow 4 \rightarrow 7$ is selected as the information transmission path of the node 1 and the node 7 , so the path $\mathrm{e} 2 \mathrm{e} 12 \mathrm{e} 9$ is the optimal path. For comparative analysis, we use Dijkstra's algorithm to calculate the above example. The simulation results of delay, reliability and bandwidth are shown in Table III.

TABLE III. COMPARISON RESULTS OF TRANDITIONAL ALGORITHM AND ALGORITHM IN THE PAPER

\begin{tabular}{|c|c|c|}
\hline Algorithm & $\begin{array}{c}\text { Time } \\
\text { Delay(ms) }\end{array}$ & $\begin{array}{c}\text { Bandwidth( } \\
\text { Mbps) }\end{array}$ \\
\hline $\begin{array}{c}\text { Dijkstra } \\
\text { Algorithm }\end{array}$ & 4.1016 & 5 \\
\hline $\begin{array}{c}\text { Algorithm in the } \\
\text { paper }\end{array}$ & 4.1018 & 8 \\
\hline
\end{tabular}

As can be seen from Table III, the delays of the above two algorithms are similar. Because Dijkstra algorithm requires only the shortest path length and does not consider other factors, the reliability calculated by this algorithm is 0.0057 higher than the reliability of Dijkstra algorithm. Meanwhile, the bandwidth is better than Dijkstra algorithm to calculate the bandwidth of the path. The results show that the proposed algorithm outperforms the Dijkstra algorithm in the routing of wide area protection communication systems.

The above analysis shows that the proposed algorithm can meet the requirements of reliability, real-time and bandwidth of the wide area communication, and can achieve the goal of maximizing the reliability and bandwidth of the information transmission path and guarantee the correct and real-time transmission of information.

\section{CONCLUSION}

Aiming at the performance requirements of wide area information transmission for communication networks, this paper proposes a path selection method to meet the requirements of reliability, delay and bandwidth optimization. A mathematical model and a solution algorithm are established. The above examples and results show that this algorithm can achieve the goal of low latency, high bandwidth requirement and maximum reliability. This method can also be used in the path reselection in the case of information transmission path failure, which improves the reliability of wide area communication network, guarantees the real-time transmission of information and reduces the congestion of information transmission.

\section{ACKNOWLEDGMENT}

This work has been supported by State Grid Corp Science and Technology Project: Research on Key Technology of 'IP+Optical' Orchestrator for Power Service.

\section{REFERENCES}

[1] Guerin R A, Orda A, Williams D. QoS routing mechaisms and OSPF extensions[C]. IEEE Global Communications Conference, 1997: 19031908 . 
[2] Liu Chuan, Li Binglin, Lou Zheng, et al. Analysis on Time Delay Performance of SDN Controller Supporting Power Service Planning[J]. Automation of Electric Power System, 2017, 41(17), 142-147.

[3] V Terzija, G Valverde, D Cai, etc. Wide-AreaMonitoring, Protection, and Control of Future Electric Power Networks[J].2011, Vol. 99, No. 1: 80-93

[4] Zhang Lei, Zhan Zhenbin, Wei Luping, et al. Test and Analysis of Piecewise Delay in Wide Area Measurement System.[J]. Automation of Electric Power System, 2016, 40(6), 101-106.

[5] Han D, Chung J M. Self-Similar Traffic End-to-End Delay Minimization Multipath Routing Algorithm[J]. IEEE Communications Letters, 2014, 18(12): 2121-2124.

[6] He Linbo, Jiang Dingde, Zhong Weijia, et al. A Routing Optimization Algorithm Based on Energy Efficiency and Frequency Effect[J]. Journal of Electronics, 2016,44 (10): 2314-2322.

[7] Jia Huibin, Xue Kaifu, Ma Jing, et al. Improved Ant Colony Algorithm for Multi-path Routing Selection in Wide-area Protection Communication System[J]. Automation of Electric Power System. 2016,40(22): 22-26. 